\title{
Ant-Colony-Based Multiuser Detection for MC DS-CDMA Systems
}

\author{
Chong Xu, Lie-Liang Yang and Lajos Hanzo \\ School of ECS., Univ. of Southampton, SO17 1BJ, UK. \\ Tel: +44-23-80-593 125, Fax: +44-23-80-593 045 \\ Email: $\{$ cx05r, lly, lh\}@ecs.soton.ac.uk, http://www-mobile.ecs.soton.ac.uk
}

\begin{abstract}
In this contribution we present a novel ant colony optimization (ACO) based multi-user detector (MUD) designed for synchronous multi-carrier direct sequence code division multiple access (MC DSCDMA) systems. The operation of the ACO-based MUD is based on the behaviour of the ant colony in nature. The ACO-based MUD aims for achieving the same bit-error-rate (BER) performance as the optimum maximum likelihood (ML) MUD, without carrying out an exhaustive search of the entire MC DS-CDMA search space constituted by all possible combinations of the received multi-user vectors. We will demonstrate that the system is capable of supporting almost as many users as the number of chips in the spreading sequence, while searching only a small fraction of the entire ML search space. It will also be demonstrated that the number of floating point operations per second is a factor of $10^{8}$ lower for the proposed ACO-based MUD than that of the ML MUD, when supporting $K=32$ users in a MC DS-CDMA system employing 31-chip Gold codes as the T-domain spreading sequence.
\end{abstract}

\section{INTRODUCTION}

Multi-carrier direct sequence code division multiple access (MC DS-CDMA) is widely recognized as a high-flexibility multiple-access scheme. It is also capable of providing a high degree of freedom for system designers and for channel-adaptive reconfiguration, when compared to both single-carrier DS-CDMA and frequency (F)domain spread multicarrier CDMA (MC-CDMA) operating without T-domain spreading [1]-[3]. In [4]-[6] the authors have proposed and investigated a MC DS-CDMA system, which employed orthogonal Walsh Hadamad codes as its T-domain spreading sequence, combined with multiple base-station (BS) antenna arrays employed for the sake of achieving either receive diversity [4] in the uplink (UL) or transmit diversity [5], [6] in the downlink (DL). A low-complexity single user detector (SUD) based on a filter matched to the spreading code of the desired user was used in the above contributions. This SUD scheme is optimal in terms of its achievable bit error rate (BER) performance versus the signal-to-noise ratio (SNR), as a benefit of the spreading codes's orthogonality in the T-domain. However, the orthogonality of the spreading codes is often destroyed by the dispersive channel.

As a design alternative, in this paper, non-orthogonal codes are employed, resulting in multi user interference (MUI), which requires the employment of multi user detection (MUD) [3], [7]. The optimal maximum likelihood (ML) MUD carries out an exhaustive search for all the legitimate combinations of the transmit symbols of all the users. Natrually, this technique has a complexity that increases exponentially with the number of users, as well as with the number of bits per symbol, which motivates the development of reducedcomplexity near-optimal MUDs. For instance, genetic algorithms (GA) [3], [8]-[10], evolutionary programming [11], particle swarm optimization [12], ant-colony optimization (ACO) [13]-[15], sphere decoding [16], [17] and Markov-Chain Monte-Carlo (MCMC) [18] aided detectors have found favour in low-complexity near-optimum MUDs.

Acknowledgements: The financial support of the EPSRC, UK and that of the EU under the auspicies of the PHOENIX and NEWCOM projects is gratefully acknowledged.

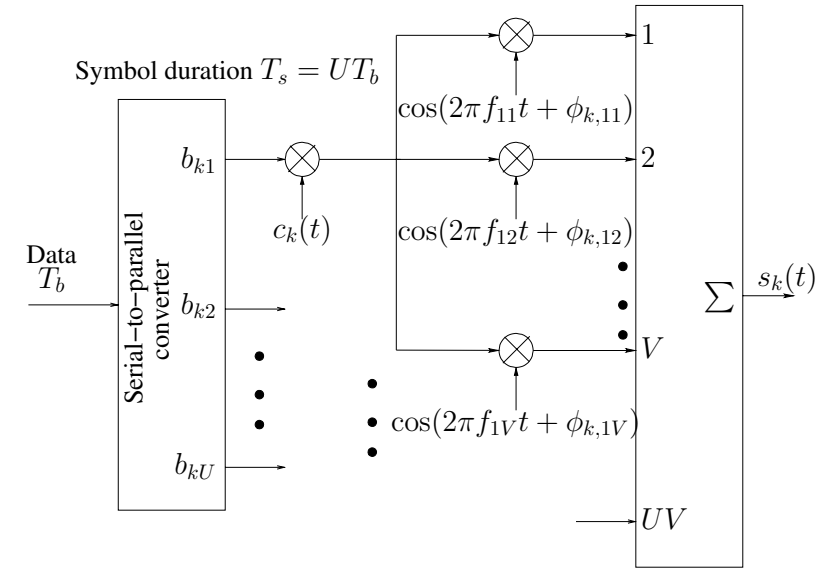

Fig. 1: The $k$ th user's uplink transmitter schematic for the generalized multicarrier DS-CDMA system.

In this paper, we present a novel low-complexity ACO based MUD designed for the synchronous MC DS-CDMA uplink. The ACO technique has been shown to outperform GAs in some Nondeterministic Polynomial time (NP)-complete optimization problems, such as the travelling salesman problem [14]. Moreover, according to [19]-[22], the ACO based MUDs are capable of achieving a lower BER and a lower complexity than the GA-based MUDs in wireless communication systems. In this contribution we extend the ACO based single-carrier DS-CDMA system's MUD of [22] to a multicarrier DS-CDMA system scenario. The complexity of both the ACO based MUD and that of some traditional detectors designed for MC DS-CDMA are investigated.

The rest of this paper is organized as follows. The MC DS-CDMA system model as well as the matched filter's (MF) output will be characterized in Section II. In Section III, the ACO algorithm will be detailed. Both the achievable BER performance and the complexity imposed will be quantified in Section IV. Finally, we will conclude our discourse in Section V.

\section{SySTEM DESCRIPTION}

\section{A. Transmitter Model}

In this subsection, the generalized MC DS-CDMA system of Fig. 1 [2], [23], [24] is reviewed. At the transmitter side, the binary data stream having a bit duration of $T_{b}$ is Serial-to-Parallel (S/P) converted to $U$ parallel sub-streams. The new bit duration of each sub-stream, which we refer to as the symbol duration, becomes $T_{s}=U T_{b}$. After $\mathrm{S} / \mathrm{P}$ conversion, each substream is spread using an $N$-chip DS spreading sequence waveform $c_{k}(t)$. Then, the DS spread signal of the $u$ th sub-stream, where we have $u=1,2, \ldots, U$, 
simultaneously modulates a group of $V$ parallel subcarrier frequencies $\left\{f_{u 1}, f_{u 2}, \ldots, f_{u V}\right\}$ using Binary Phase Shift Keying (BPSK). Thus the transmitted signal on the $u v$ th subcarrier of user $k$ can be expressed as

$$
s_{k, u v}(t)=\sqrt{\frac{2 P}{V}} b_{k u}(t) c_{k}(t) \cos \left(2 \pi f_{u v} t+\phi_{k, u v}\right),
$$

where $P / V$ represents the transmitted power of each subcarrier and $P$ is the transmitted power corresponding to each bit. Furthermore, $\left\{b_{k u}(t)\right\},\left\{f_{u v}\right\}$ and $\left\{\phi_{k, u v}\right\}$ represent the subcarrier data streams, the subcarrier frequency set and the phase angles introduced in the carrier modulation process. A total of $U V$ number of subcarriers are required in the MC DS-CDMA system considered and the $U V$ number of subcarrier signals are superimposed on each other in order to form the complex-valued modulated signal. Therefore, the transmitted signal of user $k$ can be expressed as

$$
s_{k}(t)=\sum_{u=1}^{U} \sum_{v=1}^{V} \sqrt{\frac{2 P}{V}} b_{k u}(t) c_{k}(t) \cos \left(2 \pi f_{u v} t+\phi_{k, u v}\right) .
$$

\section{B. Receiver Model}

We assume that $K$ synchronous MC DS-CDMA users are supported with the aid of a single receive antenna at the base-station (BS). Furthermore, we assume that the modulated signal of each subcarrier is orthogonal to each other. In this case, we can consider the received signal subcarrier by subcarrier. Thus the $\left(N_{c} \times 1\right)$ dimensional received signal vector containing the signals of all the $K$ users associated with the $u v$ th subcarrier can be expressed as

$$
\mathbf{r}_{u v}=\mathbf{C H}_{u v} \xi \mathbf{b}_{u}+\mathbf{n}_{u v},
$$

where $\xi=\sqrt{P / V}$ and $\mathbf{C}$ represents the $\left(N_{c} \times K\right)$-dimensional spreading code matrix. Furthermore, $\mathbf{H}_{u v}$ is a $(K \times K)$-dimensional matrix, where the diagonal elements of $\mathbf{H}_{u v}$ represent the SpatioTemporal Channel Impulse Responses (ST-CIR) of all the users modulating the $u v$ th subcarrier, which is formulated as $\mathbf{H}_{u v}=$ $\operatorname{diag}\left(h_{u v, 1}, h_{u v, 2}, \cdots, h_{u v, K}\right)$, where $h_{u v, k}$ is the complex-valued fading gain of the $u v$ th subcarrier ST-CIR connecting the $k$ th user and the BS. Finally, $\mathbf{b}_{u}$ is the $K$-user transmit signal vector of the $u$ th bit, which can be expressed as $\mathbf{b}_{u}=\left[b_{1 u}, b_{2 u}, \cdots, b_{K u}\right]^{T}$ and $\mathbf{n}_{u v}^{\left(n_{r} l\right)}$ is the $\left(N_{c} \times 1\right)$-dimensional additive white Gaussian noise (AWGN) vector, where each element has a zero mean and a variance of $2 N_{0}$. For simplicity, we assume that the ST-CIR are perfect known at the BS. It can be shown that the MRC based MF's output vector corresponding to the $u v$ th subcarrier of all the $K$ users can be expressed as

$$
\begin{aligned}
\mathbf{y}_{u v} & =\left(\mathbf{C} \mathbf{H}_{u v}\right)^{H} \mathbf{r}_{u v} \\
& =\mathbf{H}_{u v}^{H} \mathbf{C}^{T} \mathbf{C} \mathbf{H}_{u v} \xi \mathbf{b}_{u}+\mathbf{H}_{u v}^{H} \mathbf{C}^{T} \mathbf{n}_{u v} \\
& =\mathbf{R}_{u v} \xi \mathbf{b}_{u}+\tilde{\mathbf{n}}_{u v},
\end{aligned}
$$

where we have

$$
\mathbf{R}_{u v}=\mathbf{H}_{u v}^{H} \mathbf{C}^{T} \mathbf{C} \mathbf{H}_{u v}
$$

and each element in $\tilde{\mathbf{n}}_{u v}$ has a mean of zero and a variance of $2 N_{0}$.

As seen in Fig. 2, all the $V \cdot K$ number of MF's outputs $\left\{y_{1, u 1}, \ldots\right.$, $\left.y_{1, u V}, \ldots, y_{K, u 1}, \ldots, y_{K, u V}\right\}$ related to the $u$ th substream will be input to the $u$ th ACO-based MUD that will generate the $(K \times 1)$ dimensional estimate vector $\hat{\mathbf{b}}_{u}$ for $\mathbf{b}_{u}$, which comprises the transmit signals of all the $K$ users mapped to the $u$ th substream.

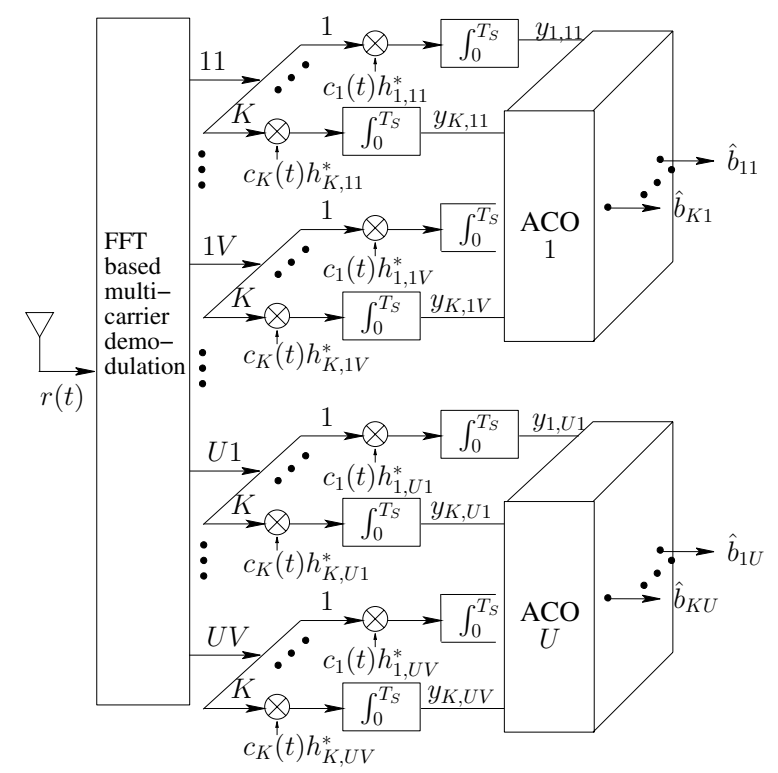

Fig. 2: Uplink receiver block diagram of the generalized MC DSCDMA system considered for all the $K$ users when employing the maximum likelihood detector or the ACO-based MUD.

\section{Ant Colony Optimization Based Multiuser Detector}

Remembering that the different subcarrier signals are orthogonal, the $U$ number of ACO-based MUDs may operate in parallel without interfering with each other. In each of the $U$ ACO-based MUDs, there is a $(2 \times K)$ matrix or so-called route-table, as shown below,

$$
\begin{array}{c|c|c|c|c|}
\multicolumn{2}{c}{1} & \multicolumn{1}{c}{2} & \cdots & \multicolumn{1}{c}{K} \\
\cline { 2 - 5 } 1 & b_{1}=+1 & b_{2}=+1 & \cdots & b_{K}=+1 \\
\cline { 2 - 5 } 2 & b_{1}=-1 & b_{2}=-1 & \cdots & b_{K}=-1 \\
\cline { 2 - 5 } & & &
\end{array}
$$

which represents the two possible choices for the bits $\hat{b}_{k u}$ of each of the $K$ users.

The ACO algorithm [19]-[22] is based on the foraging behavior of the ant colony in nature. Every ant leaves pheromone along the route from the formic nest to a certain remote source of food. Therefore, the shorter the route, the more the pheromone. As a beneficial effect of the pheromone, the ants about to set out from the nest later are more likely to choose the particular route marked by a higher concentration of pheromone. As a result, most ants will choose the shortest route from the nest to the source of food. We assume that there is a total of $N$ iterations, when searching for the best route in each ACO-based MUD. More specifically, in each of the $N$ iterations, $M$ ants work their way through the $K$ columns of the matrix seen in the route-table, in order to produce $M$ number of binary $K$ user vectors, serving as the $M$ candidate vectors of $\hat{\mathbf{b}}_{u}$. Borrowing from the above mechanism, the flow chart of an ACO-based MUD algorithm is depicted in Fig. 3. At this stage, it is worth relating the ACO-based MUD philosophy to another random guided algorithm, namely to the family of GA aided MUDs applied in the context of both single- and multi-carrier CDMA in [3], [9]. More specifically, in GA-parlance we may commence the search for a near-ML $K$ user bit-vector from a so-called initial population of $M K$-bit vector and generate $N$ consecutive so-called generations of $K$-user bitvectors, each generation containing $M$ candidate vectors. The $K$-bit candidate vecotrs of the initial population are modified with the aid of random guided bit-mutations by exchanging bits between the highprobability $K$-bit vectors using operations referred to as cross-over etc. Following this brief analogy, let us now return to the ACO-aided 


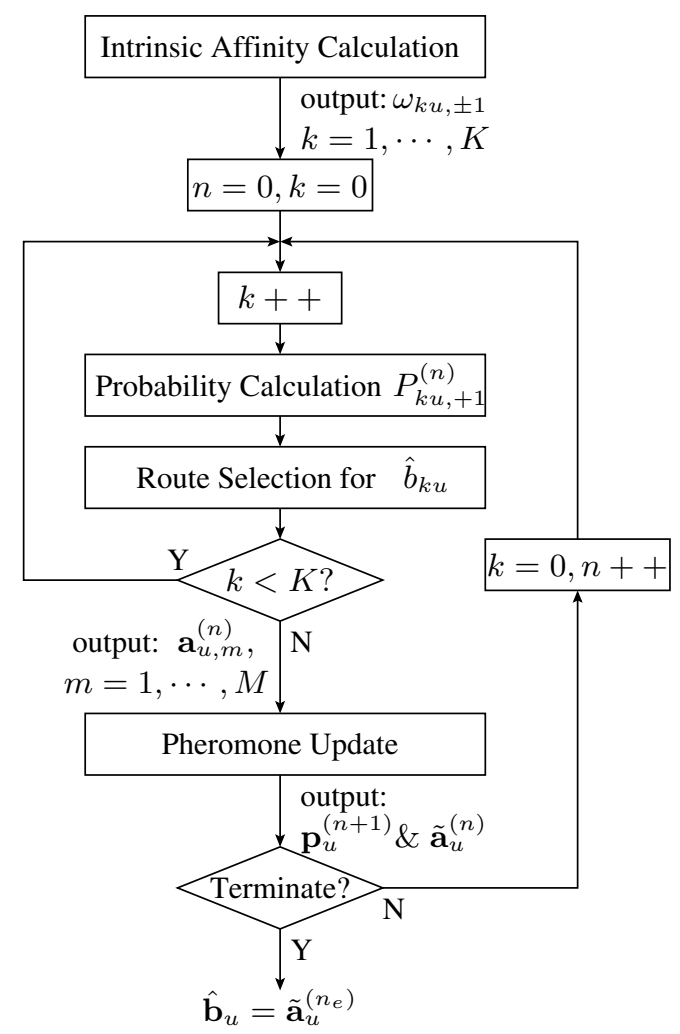

Fig. 3: Flow-chart of each of the $U$ ACO-based MUDs.

MUD.

\section{A. Calculation of the Intrinsic Affinity}

Let us extend our ant-analogy a little further. In addition to following the high-probability routes marked by a high concentration of pheromone, the ants prefer a flat and comfortable route to the source of food. In MUD-parlance, a particular bit of the $K$-bit vector has a high user-specific LLF value, if it represents a confident bit-decision. In ACO jargon, a high user-specific-LLF-based user-bit corresponds to a less arduous passage of the ants, hence the corresponding bitvalue is likely to be confirmed at a high pheromone-level by several of the $M$ ants. The flow chart of the instrinsic bit-affinity calculation is shown in Fig. 4.

More quantitatively, let us first of all introduce the so-called desirability function, which can be expressed as [25]:

$$
d_{k u, \pm 1}=1+\exp \left[-l_{k u}( \pm 1)\right]
$$

where $l_{k u}( \pm 1)$ represents the LLF related to the $k$ th user's bit $b_{k u}=$ \pm 1 in the $u$ th substream. In our MC DS-CDMA system, the LLF is given by the superposition of the LLFs associated with all the $V$ subcarriers $\left\{f_{u 1}, f_{u 2}, \ldots, f_{u V}\right\}$ invoked for transmitting $b_{k u}$. This is formulated as

$$
l_{k u}( \pm 1)=\sum_{v=1}^{V} l_{k, u v}( \pm 1)
$$

and the user-specific LLF of $b_{k u}= \pm 1$ associated with the $u v$ th subcarrier can be expressed as

$$
l_{k, u v}( \pm 1)= \pm 2 \sqrt{\frac{P}{V}} \Re\left(y_{k, u v}\right)-\frac{P}{V} R_{u v, k k},
$$

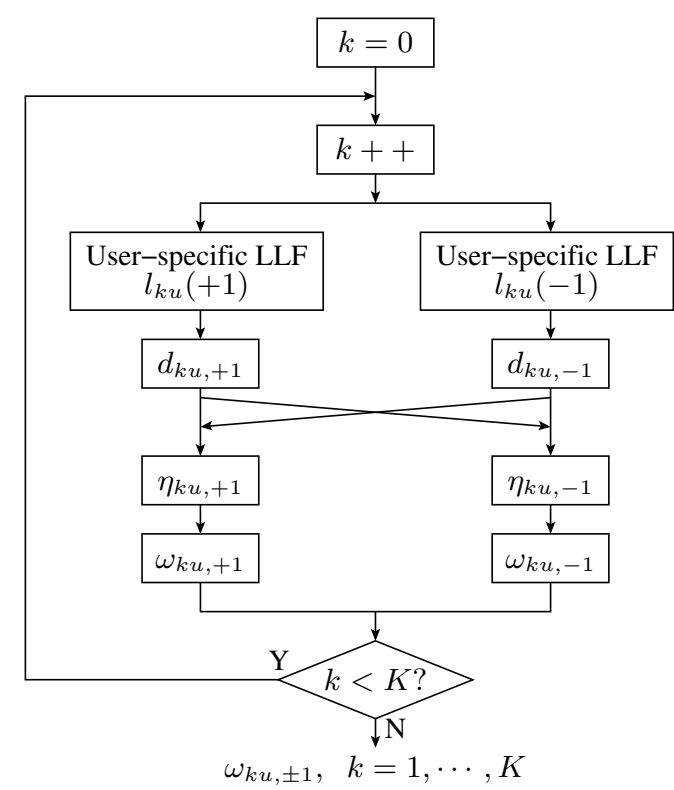

Fig. 4: Detailed flow-chart of the 'Intrinsic Affinity Calculation' block in Fig. 3.

where $R_{u v, k k}$ represents the $k$ th diagonal element of $\mathbf{R}_{u v}$. Given the desirability function of (6), the sum of its two legitimate values is nomalized according to [22]

$$
\eta_{k u, \pm 1}=\frac{\sum_{i \in\{+1,-1\}} d_{k u, i}}{d_{k u, \pm 1}}
$$

Given the normalized desirability function of 9 , the intrinsic bitinfinity of the $k$ th user in the $u$ th substream may be expressed as

$$
\omega_{k u, \pm 1}=\eta_{k u, \pm 1}^{\beta},
$$

where $\beta$ is tunable weighting or de-weighting parameter.

\section{B. Probability Calculation and Route Selection}

Having completed the intrinsic affinity calculation step of Fig. 3, let us now consider the probability-calculation and route-selection operations of Fig. 3. When considering the two choices of +1 or -1 for user $k, \bar{M}_{k u}^{(n)}$ number of ants will choose +1 and all the other $\left(M-\bar{M}_{k u}^{(n)}\right)$ ants will opt for -1 . The specific number of ants $\bar{M}_{k u}^{(n)}=\Gamma\left[M P_{k u,+1}^{(n)}\right]$ is based on the probability $P_{k u,+1}^{(n)}$ calculated by combining the intrinsic affinity of +1 or -1 and its associated pheromone intensity, while $\Gamma\left[M P_{k u,+1}^{(n)}\right]$ represents the rounded-down integer version of $\left(M P_{k u,+1}^{(n)}\right)$. More explicitly, the probability of opting for +1 as the esitmated version of $b_{k u}$ during the $n$th iteration can be expressed as [22]

$$
P_{k u,+1}^{(n)}=\frac{\mu_{k u,+1}^{(n)} \omega_{k u,+1}}{\sum_{i \in\{+1,-1\}} \mu_{k u, i}^{(n)} \omega_{k u, i}},
$$

where $\mu_{k u, \pm 1}^{(n)}=\left[p_{k u, \pm 1}^{(n)}\right]^{\alpha}$ represents the effect of the pheromone intensity, while $\omega_{k u, \pm 1}$ is the intrinsic affinity introduced in the last subsection. Furthermore, $\alpha$ is a tunable weighting coefficient and $p_{k u, \pm 1}^{(n)}$ is the specific element in the $k$ th column and in the 1 st or 2 nd row of the $(2 \times K)$-dimensional matrix $\mathbf{p}_{u}^{(n)}$ representing the pheromone intensity corresponding to the bit-elements in the routetable. 


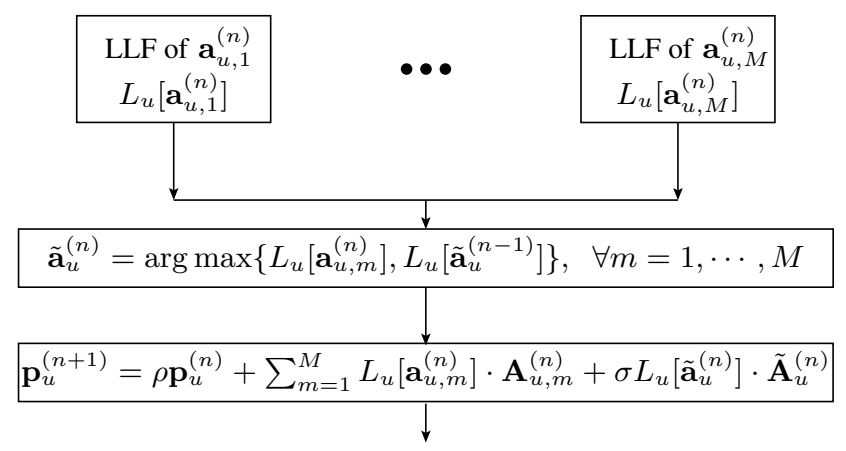

Fig. 5: Detailed flow-chart of the 'Pheromone Update' block in Fig. 3.

\section{Pheromone Update}

Let us now continue by considering the pheromone update block of Fig. 3. As observed in the foraging behavior of the ants in nature, every ant in the ACO-aided MUD will leave a certain amount of pheromone along the route it follows. The more ants pursue a particular route, the more pheromone will be assigned to the route. Analogously to the ants in nature which use the distance as their route-quality criterion, the quality of the route in our algorithm is quantified in terms of its LLF.

Based on our above discussions, the amount of pheromone assigned to each bit constituting a specific route is set to the LLF of the route. The corresponding flow chart of the pheromone update procedure is outlined in Fig. 5. More explicitly, the amount of pheromone assigned to a particular route pursued by the $m$ th ant during the $n$th iteration is formulated as

$$
\Delta \mathbf{p}_{u, m}^{(n)}=L_{u}\left[\mathbf{a}_{u, m}^{(n)}\right] \cdot \mathbf{A}_{u, m}^{(n)},
$$

where $\mathbf{a}_{u, m}^{(n)}$ represents the specific $K$-user vector produced by the $m$ th ant during the $n$th iteration and $L_{u}\left[\mathbf{a}_{u, m}^{(n)}\right]$ represents the LLF related to the $K$-user vector $\mathbf{b}_{u}=\mathbf{a}_{u, m}^{(n)}$ in the $u$ th substream, while $\mathbf{A}_{u, m}^{(n)}$ is the $(2 \times K)$-dimensional matrix representation of $\mathbf{a}_{u, m}^{(n)}$. For instance, if we have a 4 -user vector produced by the $m$ th ant given by $\mathbf{a}_{u, m}^{(n)}=[+1,-1,+1,+1]^{T}$, we arrive at

$$
\mathbf{A}_{u, m}^{(n)}=\left[\begin{array}{llll}
1 & 0 & 1 & 1 \\
0 & 1 & 0 & 0
\end{array}\right]
$$

Furthermore, an extra amount of pheromone quantified by $\sigma \cdot L_{u}\left[\tilde{\mathbf{a}}_{u}^{(n)}\right]$ is assigned to a route leading to the most likely $K$-user vector $\tilde{\mathbf{a}}_{u}^{(n)}$ found so far, where $\sigma$ represents the weighting factor of the pheromone assigned to a meritorious $K$-user vector. However, there is also a so-called evaporation rate [19]-[22] $\rho$, allowing the previously assigned pheromone to evaporate, hence creating chances for the ants to find new routes during the next cycle that may be more meritorious than the best route found so far. Finally, the pheromone matrix generated for the $(n+1)$ st iteration can be obtained by [22]

$$
\mathbf{p}_{u}^{(n+1)}=\rho \mathbf{p}_{u}^{(n)}+\sum_{m=1}^{M} \Delta \mathbf{p}_{u, m}^{(n)}+\sigma L_{u}\left[\tilde{\mathbf{a}}_{u}^{(n)}\right] \cdot \tilde{\mathbf{A}}_{u}^{(n)}
$$

where $L_{u}\left[\tilde{\mathbf{a}}_{u}^{(n)}\right]$ represents the LLF related to the $K$-user vector $\mathbf{b}_{u}=$ $\tilde{\mathbf{a}}_{u}^{(n)}$ in the $u$ th substream.

In our MC DS-CDMA system, the calculation of the LLF value formulated in (12) and (14) and related to the $K$-user vector $\mathbf{b}_{u}$ being a specific vector $\dot{\mathbf{b}}_{i} \in \mathcal{B}_{K}$, where $\mathcal{B}_{K}$ contains all the $2^{K}$ possible combinations for the $K$-user transmit vector, is given by the superposition of the LLFs associated with all the $V$ subcarriers $\left\{f_{u 1}, f_{u 2}, \ldots, f_{u V}\right\}$ invoked for transmitting $\mathbf{b}_{u}$. This is formulated as

$$
L_{u}\left(\dot{\mathbf{b}}_{i}\right)=\sum_{v=1}^{V} L_{u v}\left(\dot{\mathbf{b}}_{i}\right), \quad \dot{\mathbf{b}}_{i} \in \mathcal{B}_{K}
$$

and the LLF of $\mathbf{b}_{u}=\dot{\mathbf{b}}_{i}$ associated with the $u v$ th subcarrier can be expressed as

$$
\begin{aligned}
L_{u v}\left(\dot{\mathbf{b}}_{i}\right)=2 \sqrt{\frac{P}{V}} \Re\left(\dot{\mathbf{b}}_{i}^{T} \mathbf{y} u v\right) & -\frac{P}{V} \dot{\mathbf{b}}_{i}^{T} \mathbf{R}_{u v} \dot{\mathbf{b}}_{i}, \\
i & =1,2, \ldots, 2^{K} .
\end{aligned}
$$

\section{Termination Condition}

The optimization procedure of the $u$ th ACO-based MUD designed for finding the near-ML $K$-bit vector $\hat{\mathbf{b}}_{u}$ will terminate at the $n_{e}$ th iteration, if either all the ants produce the same $K$-user vector estimate during the $n_{e}$ th cycle, or all the $N$ number of affordable ACO iterations have been carried out, i.e. we have $n_{e}=N$. Then the MUD's output $\hat{\mathbf{b}}_{u}$ is given by

$$
\hat{\mathbf{b}}_{u}=\tilde{\mathbf{a}}_{u}^{\left(n_{e}\right)} \text {. }
$$

Let us now consider both the achievable BER performance and the complexity imposed, when carefully selecting the algorithm's parameters in the next section.

\section{Simulation Result}

In this section both the attainable BER performance and the complexity of the uplink MC DS-CDMA system using the ACO-based MUD is investigated, when assuming that each subcarrier signal experiences flat Rayleigh fading. In our simulations we assumed that the number of subcarriers varies from $V=1$ to $V=128$ and that the T-domain spreading sequences were the $N_{c}=31$-chip Gold codes. The BER performance and the complexity of the proposed ACObased MUD employed in the MC DS-CDMA UL is also compared to that of the MRC-based correlation detector and to that of the ML MUD detailed in Section II.

Throughout our simulations, the initial pheromone level of $p_{k u, \pm 1}^{(1)}=0.01$ was used for all the $u$ th, $u=1 \ldots U$, bit of the $k$ th, $k=1 \ldots K$ user in conjunction with $\rho=0.5, M=10, N=10$, $\alpha=1, \beta=6$ and $\sigma=8$.

Fig. 6 shows the BER performance versus signal-to-noise ratio (SNR) performance of the MRC-based correlation detector, the ML MUD and the ACO-based MUD, which was introduced in Sections II and III. Again, the number of subcarriers was varied for $V=1, \ldots, 128$. Both the uplink MC DS-CDMA system employing the MRC-based correlation detector and the ACO-based MUD are capable of supporting $K=32$ users, which is on the order of $O\left(2^{32}\right)$. By contrast, the MC DS-CDMA system employing the ML MUD has an excessive complexity for $K=32$ users. Fig. 7 shows the complexity versus the number of users at SNR $=10 \mathrm{~dB}$ for the MRC-based correlation detector, for the ML MUD and the ACObased MUD along with a different number of subcarriers varing from $V=1, \ldots, 128$.

As can be seen in Fig. 6, the ACO-based MUD is capable of approaching the BER performance of the ML MUD regardless of the number of subcarriers. On the other hand, as shown in Fig. 7, regardless of the number of subcarriers, the complexity of the ACObased MUD is similarly low to that of the MRC-MF and is only a fraction of that of the ML MUD, especially when the number of users is high. For example, when the number of users is $K=32$, the complexity of the ACO-based MUD is deemed to be a factor of $10^{8}$ lower than that of the MLD. 


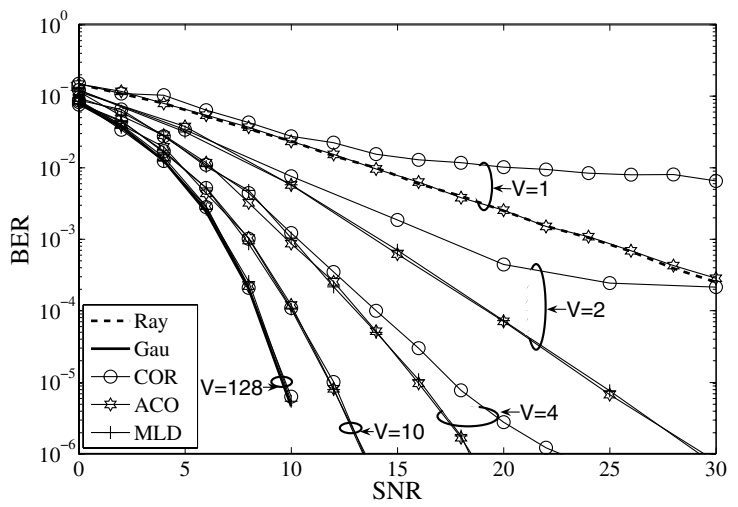

Fig. 6: BER versus SNR performance of the uplink MC DS-CDMA system considered using $V=1,2,4,10,128$ subcarriers and $N_{c}=$ 31-chip Gold codes for T-domain spreading. Both the ACO-based MUD and the correlation detector support $K=32$ users, while the ML MUD has an excessive complexity.

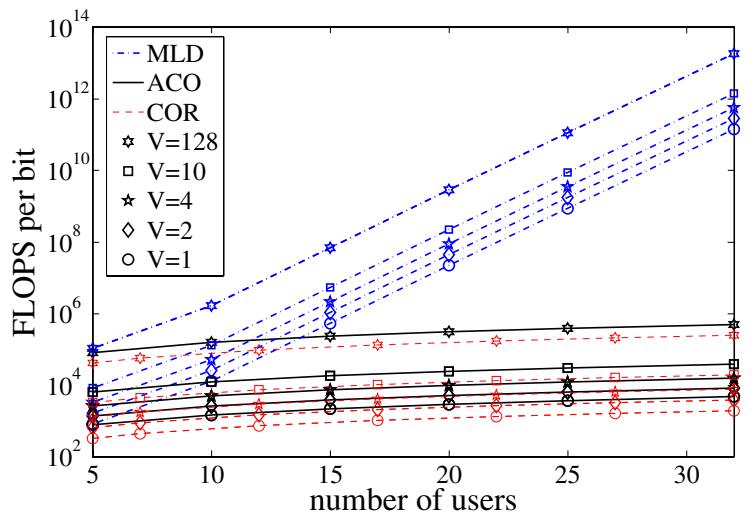

Fig. 7: Complexity per user per bit versus the number of users supported in the uplink of the MC DS-CDMA system considered using $V=1,2,4,10,128$ subcarriers and $N_{c}=31$-chip Gold codes for T-domain spreading.

\section{CONCLUSION}

We have investigated an ACO-based MUD designed for the uplink of an MC DS-CDMA system. Our simulation results show that regardless of the number of subcarriers, the ACO-based MUD approaches the BER performance of that of the ML MUD in the proposed MC DS-CDMA system supporting $K=32$ users with the aid of length-31 Gold codes used as the T-domain spreading sequences. Our simulation results also show that the proposed ACObased MUD employed in the MC DS-CDMA system considered is capable of supporting the same number of users at the number of chips in the Gold code in the vicinity of the ML MUD's BER performance at a complexity, which is similar to that of the MF detector.

\section{REFERENCES}

[1] L.-L. Yang and L. Hanzo, "Multicarrier DS-CDMA: A multiple-access scheme for ubiquitous broadband wireless communications," IEEE Communications Magazine, vol. 41, no. 10, pp. 116-124, October 2003.
[2] L.-L. Yang and L. Hanzo, "Performance of generalized multicarrier DS-CDMA over Nakagami- $m$ fading channels," IEEE Transactions on Communications, vol. 50, pp. 956 - 966, June 2002.

[3] L. Hanzo, L.-L. Yang, E.-L. Kuan and K. Yen, Single- and Multi-Carrier DS-CDMA Multi-User Detection, Space-Time Spreading, Synchronisation and Standards. Chichester, UK: John Wiley and Sons, Ltd, 2003.

[4] B. Hu, L.-L. Yang and L. Hanzo, "Performance of the smart antenna aided multicarrier DS-CDMA uplink," in IEEE 60th Vehicular Technology Conference. IEEE, September 2004, pp. 191 - 195.

[5] B. Hu, L.-L. Yang and L. Hanzo, "Performance of the smart antenna aided generalized multicarrier DS-CDMA downlink using both timedomain spreading and steered space-time spreading," IEEE 62nd Vehicular Technology Conference, vol. 1, no. 28-25, pp. 458-462, Sept. 2005.

[6] C. Xu, B. Hu, L.-L. Yang and L. Hanzo, "Performance of multi-antenna array assisted MC DS-CDMA using downlink preprocessing based on singular value decomposition," in Proceedings of IEEE Vehcular Technology Conference, 2007, pp. 1936-1940.

[7] S. Verdu, Multiuser Detection. Cambridge University Press, 1998

[8] T.-C. Fogarty, "Using the genetic algorithm to adapt intelligent systems," IEE Colloquium on symbols versus Neurons, vol. 12, pp. 4/1-4/4, October 1990

[9] Y. Kai and L. Hanzo, "Genetic algorithm assisted joint multiuser symbol detection and fading channel estimation for synchronous CDMA systems," IEEE Journal on Selected Areas in Communications, Vol. 19, pp. 985-998, June 2001.

[10] M. Jiang, S.-X. Ng and L. Hanzo, "Hybrid iterative multiuser detection for channel coded space division multiple access OFDM systems," IEEE Transactions on Vehicular Technology, vol. 55, pp. 115-127, Jan. 2006.

[11] D. Fogel, "What is evolutionary computation?," IEEE Spectrum, vol. 37, pp. 28-32, Feburary 2000.

[12] J. Kennedy and R. Eberhart, "Particle swarm optimization," IEEE International Conference on Neural Networks, vol. 4, pp.1942-1948, December 1995.

[13] M. Dorigo, L. Gambardella, M. Middendorf and T. Stutzle, "Guest editorial: special section on any colony optimization," IEEE Transactions on Evolutionary Computation, vol. 6, pp.317-319, August 2002.

[14] M. Dorigo and L. Gambardella, "Ant colony system: a cooperative learning approach to the travelling salesman problem," IEEE Transactions on Evolutionary Computation, vol. 1, pp.53-66, April 1997.

[15] M. Dorigo and G. Di Caro, "Ant colony optimization: a new metaheuristic," Proceedings of the 1999 Congress on Evolutionary Computation, vol. 2, pp.1470-1477, July 1999.

[16] L. Hanzo and T. Keller, OFDM and MC-CDMA: A Primer. Chichester, UK: John Wiley and Sons, Ltd, 2006

[17] J. Boutrous and E. Viterbo, "Signal space diversity: A power and bandwidth efficient diversity technique for the Rayleigh fading channel," IEEE Transactions on Information Theory, vol. 44, pp.1453-1467, Jul. 1998.

[18] Z.-G. Yang, B. Lu and X.-D. Wang, "Bayesian Monte Carlo multiuser receiver for space-time coded multicarrier CDMA systems," IEEE Journal on Selected Areas in Communications, vol. 19, pp.1625-1637, Aug. 2001.

[19] S.L. Hijazi, A.J. Best B. Natarajan and S. Das, "Ant-colony based optimal MC-CDMA multiuser detector," IEEE International Conference on Wireless And Mobile Computing, Networking And Communications, vol. 1, pp.128-132, August 2005.

[20] S.L. Hijazi, B. Natarajan and S. Das, "An ant-colony algorithm for multi-user detection in wireless communication systems," Genetic and Evolutionary Computation Conference, pp.2121-2126, June 2005.

[21] S.L. Hijazi and B. Natarajan, "Novel low-complexity DS-CDMA multiuser detector based on ant colony optimization," IEEE Vehicular Technology Conference, vol. 3, pp.1939-1943, September 2004.

[22] J.-J. Lai and J.-K. Lain, "Antenna-diversity-assisted ant-colony-based multiuser detection for DS-CDMA systems," IEEE International Workshop on Cellular Neural Networks and Their Applications, vol. 3, pp.106-109, May 2005.

[23] L.-L. Yang and L. Hanzo, "Performance of generalized multicarrier DS-CDMA over using various chip waveforms," IEEE Transactions on Communications, vol. 51, pp. 748-752, May 2003.

[24] L.-L. Yang and L. Hanzo, "A unified approach to the analysis of multicarrier DS-CDMA over Nakagami- $m$ fading channels", in Proceedings of IEEE GLOBECOM, (San Antonio, Texas, USA), November 25-29, 2001.

[25] J.G. Proakis, Digital Communications Fourth Edition. McGraw-Hill, 2000. 